\title{
STUDIES OF GRAIN BOUNDARY REGIONS IN DEFORMED POLYCRYSTALLINE ALUMINUM USING SPHERICAL NANOINDENTATION
}

\section{Introduction}

Mesoscale interfaces in materials, including grain boundaries, play a significant role in determining the mechanical properties of materials. The Hall-Petch effect [1, 2], discovered over sixty years ago, provides a robust but empirical relationship between the macroscopic yield strength and average grain size in a material. However, there is still very little understanding about the precise role of grain boundaries during plastic deformation and there is no consensus on the correct physics-based model for predicting this effect [3, 4]. Moreover, the Hall-Petch effect, captures only the homogenized effect of grain boundaries on the macroscale mechanical properties of metallic materials. In other words, this simple model does not pay attention to the difference in the behavior of the different type of boundaries. Various criteria have been put forth to describe the behavior of grain boundaries based on grain boundary character [5-7], interfacial structure (CSL boundaries) [7-9], grain boundary energy (special boundaries) [10, 11], and slip transfer rules [12-14]. However, these dependencies are not yet established quantitatively. An accurate understanding of the role of grain boundaries is critical for the development of robust physics-based crystal plasticity models [15-18] to accurately predict material behavior during and following plastic deformation of polycrystalline materials.

Lack of rigorous protocols for reliably capturing the mechanical response at the submicron length scales of interest constitutes the foremost technical gap in efforts aimed at advancing the fundamental understanding of the role of grain boundaries during plastic deformation. Approaches such as in-situ testing of micro-pillars [19-22] have an inherent drawback in that these methods require sophisticated equipment and are effort intensive, and therefore cannot be used to test a large number of interfaces in a cost and time efficient manner. Alternatively, instrumented indentation testing (both nano and micro) [23-26], when combined with improved data analyses protocols provides a high throughput approach suitable for addressing this challenge. The use of nanoindentation testing for characterizing grain boundary regions has been reviewed in Ref. [27]. 
Recent advances in the analysis of spherical nanoindentation data now provide a means of extracting very reliable and repeatable measurements of local mechanical properties at a submicron length scale [28-31]. This local mechanical behavior data can be combined with local structure information obtained using complementary techniques such as orientation imaging microscopy (OIM) to further our understanding of local structure-property relationships in metallic materials and their evolution during macroscopically imposed plastic deformation. In previous work [32], we studied the evolution of slip-resistances within individual grains (i.e., in the grain interiors) during deformation in polycrystalline aluminum (Al). In this work, we have conducted a detailed study into the evolution of local mechanical properties in the grain boundary regions in plastically deformed samples of polycrystalline Al. As mentioned earlier, this information is critical to the development of physics-based multiscale models for plastic deformation in polycrystalline metals. Pathak et al. [33] have already demonstrated the viability of the combined spherical nanoindentation-OIM testing and analysis protocols in quantifying changes in mechanical properties in the grain boundary regions of a deformed $\mathrm{Fe}-3 \% \mathrm{Si}$ steel sample. In that work, three specifically selected grain boundaries were studied in 30\% deformed samples. In this work, we have systematically investigated regions around eight grain boundaries in a high purity aluminum sample deformed by plane strain compression to obtain a $20 \%$ reduction in height. The smaller strain level and the larger number of grain boundaries were selected for the present study with the specific goal of quantitatively relating salient grain boundary parameters to the measured changes in the local mechanical properties in the grain boundary regions. The salient grain boundary parameters explored in this work are the grain-tograin misorientation across the boundary and the difference in the Taylor factors. Although both parameters are related to the orientations of the grains adjoining the grain boundary, the Taylor factors provide a more direct measure of the influence of the crystal lattice orientation on the plastic flow stress, while accounting for the details of the imposed deformation mode (typically expressed as a stretching tensor). Historically, there has been a general hypothesis in prior literature that the strain hardening in the grain boundary region would be strongly influenced by the grain-to-grain misorientation across the boundary [34-36]. However, some prior studies [32, 37-39] have highlighted the possibility that the difference in the Taylor factors across the grain boundary might play a more influential role in grain boundary plasticity as it explicitly captures the relative capability of the grains involved in accommodating the imposed plastic deformation. 
In order to evaluate critically the relative roles of both these grain boundary parameters, the grain boundaries for the present study were specifically selected to include different combinations of grain-to-grain misorientation across the boundary and the difference in Taylor factors.

\section{Spherical Nanoindentation and Indentation Stress-Strain (ISS) Curves}

Nanoindentation is a versatile tool for measuring the mechanical properties from small material volumes [40]. Traditionally, indentation experiments have been carried out with sharp tips [41-43], and the values of local elastic modulus and hardness were extracted mainly from an analysis of the unloading portion of the test segment [24, 44, 45]. However, recent advances in instrumentation (e.g., the availability of the continuous stiffness measurement (CSM) [46]) have now made it possible to convert the measured load-displacement data from spherical nanoindentation into highly reproducible and consistent indentation stress-strain (ISS) curves [28]. It has been demonstrated that these new protocols produce reasonable estimates of the indentation modulus and the indentation yield strength $\left(Y_{\text {ind }}\right)$. The procedures used to convert raw indentation data into ISS curves and extract $Y_{\text {ind }}$ from the ISS data have been detailed in prior publications [28, 32, 47]. A brief overview of this strategy is provided here.

The methodology for converting raw spherical nanoindentation data into ISS curves is largely based on Hertz Theory [48-50]. The first step involves identification of the effective zero point, such that all the measured signals provide the best agreement with the Hertz theory beyond this point. If $\widetilde{P}, \tilde{h}$ and $S$ are the measured load, displacement and stiffness signals, and $P^{*}$ and $h^{*}$ denote the raw load and displacement values at the effective zero point, the expected relationship between all these variables in the initial elastic regime can be shown as [49]

$$
S=\frac{3 P}{2 h_{e}}=\frac{3\left(\tilde{P}-P^{*}\right)}{2\left(\tilde{h}-h^{*}\right)}
$$

where $h_{e}$ is the elastic indentation depth at load $P$. Linear regression can be used to determine the values of $P^{*}$ and $h^{*}$, i.e., the effective zero point [28]. The second step is to determine the radius of contact $(a)$ as $[28,49]$

$$
\begin{aligned}
& a=\frac{S}{2 E_{\text {eff }}}, \\
& \frac{1}{E_{\text {eff }}}=\frac{1-v_{s}^{2}}{E_{s}}+\frac{1-v_{i}^{2}}{E_{i}}
\end{aligned}
$$


where $E_{\text {eff }}$ is the effective stiffness of the indenter-sample system, $E$ and $v$ are the Young's modulus and Poisson's ratio, respectively, and the subscripts $s$ and $i$ refer to the sample and the indenter, respectively. As the last step, the values of indentation stress $\left(\sigma_{\text {ind }}\right)$ and indentation strain $\left(\varepsilon_{\text {ind }}\right)$ are extracted from the raw data using [28]

$$
\sigma_{\text {ind }}=\frac{P}{\pi a^{2}}, \quad \varepsilon_{\text {ind }}=\frac{4 h_{t}}{3 \pi a}
$$

It has been shown in [51] that the most reliable ISS curves for aluminum can be obtained using the CSM at $45 \mathrm{~Hz}$ oscillation frequency and a $2 \mathrm{~nm}$ oscillation amplitude. These test conditions were utilized in all of the measurements reported in this paper. From the ISS curves, $Y_{\text {ind }}$ was determined as the point of intersection between the modulus line (i.e., along the initial linear elastic portion) and the best-fit line for the stress-strain curve within the strain range of 0.0065 and 0.013 [32]. This approach was developed in our prior work to estimate $Y_{\text {ind }}$ when some small pop-ins (these are strain jumps caused by delayed activation of dislocation sources in the indentation zone, especially in the tests on annealed samples [52]) appear in the ISS curves. Although the pop-ins are almost completely absent in the deformed samples [52], the same protocols are employed consistently on both annealed and deformed samples.

\section{Materials and Methods}

High purity, polycrystalline aluminum (99.999\%) was used for this work. Pure aluminum does not exhibit annealing twins or deformation twins. This allows a focused study where dislocation slip is the only mechanism of plastic deformation [37, 38, 53]. Aluminum also exhibits low elastic anisotropy $(A=1.22)[54]$ and hence the effect of crystal orientation on the indentation modulus is rather small. A modified form of Hertz theory [55] predicts the sample indentation modulus for grains of different orientations to be in the range of 68.8 to $70.6 \mathrm{GPa}$. The surface oxide film in aluminum is extremely thin, thus making its interference with our measurements negligible.

Since we intended to interrogate regions near grain boundaries, a fully annealed, large grained sample was used for this study, so that the grain boundary segments were long enough to allow a large number of measurements while staying away from triple junctions. In order to achieve these large grains, a sample was cut out from a heavily rolled block of high purity aluminum and annealed at $640^{\circ} \mathrm{C}$ for 72 hours. The sample was then deformed by plane strain 
compression in a channel-die at room temperature to achieve $20 \%$ reduction in height. Care was taken to ensure that all sample surfaces were in contact with the die and the punch were lubricated with a $0.1 \mathrm{~mm}$ thick Teflon tape to minimize friction. The compression itself was carried out in multiple small steps and the sample was lubricated between steps. The sample was then mounted in epoxy and the surface was prepared using mechanical grinding and polishing. Since a high quality surface finish is extremely important for indentation measurements [56], electropolishing was used as the final sample preparation step. The polishing removed about $0.5 \mathrm{~mm}$ of material from the sample surface, thus eliminating any material close to the surface that may have been affected by friction.

An orientation map of the polished surface was obtained using orientation imaging microscopy (OIM), which is based on the automated indexing of electron backscatter diffraction patterns (EBSD). Bunge-Euler angles $[57,58]$ were used to denote the orientation at each point on the surface. These are an ordered set of three rotations $\left(\varphi_{1}, \Phi, \varphi_{2}\right)$ that transform the sample reference frame to the crystal reference frame. The grains on the surface were numbered for convenience and will be henceforth referred to using the assigned grain numbers. From this OIM map, eight grain boundaries were selected for this study. These were selected to include a broad range of grain boundaries, including low and high grain-to-grain misorientation angle boundaries as well as low, moderate, and high deviations in the Taylor factors of the grains on either side of the grain boundary.

With an aim to obtain better spatial resolution and also place as many indents as possible close to the grain boundary, a $20 \mu \mathrm{m}$ radius spherical indenter tip was used. Data generated in prior work [32] had quantified the effect of orientation on $Y_{\text {ind }}$ for the high purity aluminum using a $100 \mu \mathrm{m}$ radius spherical indenter tip; the same material will be used in this study. As mentioned earlier and discussed in detail in prior work [52], pop-ins (i.e., displacement jumps during an indentation test) produce a discontinuity in the ISS curves making the determination of $Y_{\text {ind }}$ difficult. Occurrence of pop-ins is related to the difficulty of establishing potent dislocation sources in the small indentation zones and is almost always observed when indentation measurements are carried out on annealed samples using indenter tips of a small radius. This was the main motivation behind the use of the larger spherical tip in prior work. As mentioned earlier, for the present study, we needed to use a smaller spherical tip to obtain a higher spatial resolution in our measurements near the grain boundary. The effect of the indenter tip size on the 
indentation response of the $20 \%$ deformed sample is shown is Figure 1 . All data used for the comparisons was obtained within the same grain. As seen from the figure, although there is a significant difference in the post elastic behavior, the values of $Y_{\text {ind }}$ are in excellent agreement in the two ISS curves. This observation is in agreement with the findings discussed in [33] regarding the effect of indenter tip size on the ISS curves. For this work, since we are interested only in the $Y_{\text {ind }}$ values, switching the indenter tip size between $20 \mu \mathrm{m}$ and $100 \mu \mathrm{m}$ did not affect the extracted values.

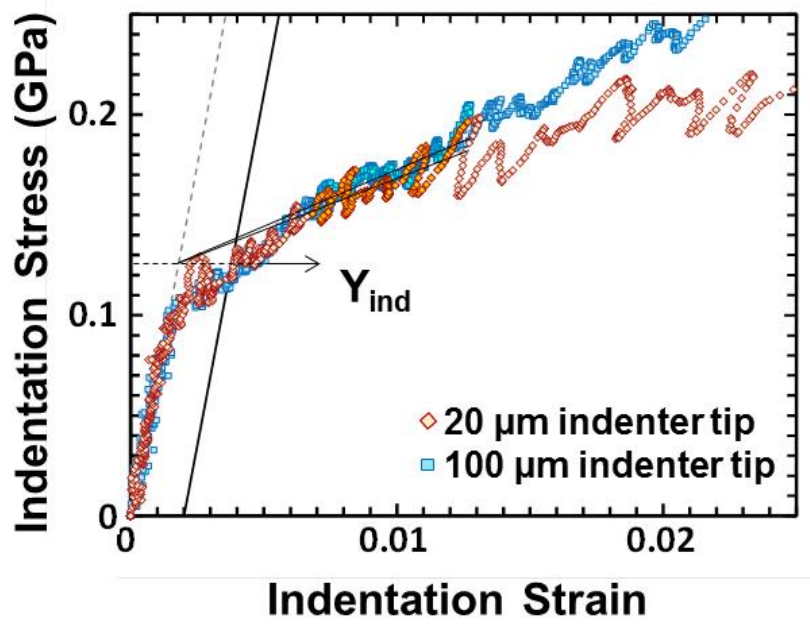

Figure 1: Representative ISS curves for tests performed with the 20um and 100um spherical indenter tip showing that the extracted $Y_{\text {ind }}$ values are not affected by the indenter tip size. Also note that the $Y_{\text {ind }}$ values extracted using the back-extrapolation method are almost the same as that produced using the $0.2 \%$ offset yield method.

Indentation tests were performed along lines inclined to the selected grain boundary at shallow angles so that a large number of measurements could be performed within a narrow region around the boundary. The long grain boundary segments, which are a result of the large grains in our samples, allowed us to place multiple rows (6-9) of indents close to the boundary. Each row contained 11 to 13 indents spaced $20 \mu \mathrm{m}$ apart. This resulted in approximately 60 to 100 indents across each grain boundary. The large number of measurements within a narrow grain boundary region allows us to ensure that the trends observed in our investigations can be distinguished from the inherit noise in the nanoindentation response. It should be noted that such a high number of data-points would be almost impossible to obtain 
using other small-scale mechanical testing techniques such as micro-pillar testing. High resolution OIM maps as well as optical micrographs were used to image the indents near the grain boundaries of interest. These were used to extract crystallographic information in the vicinity of the grain boundary as well as accurately determine the position of the indents with respect to the grain boundary. The perpendicular distance from the center of each indent to the grain boundary was calculated from these micrographs, and will hence forth be referred to as the distance from the grain boundary.

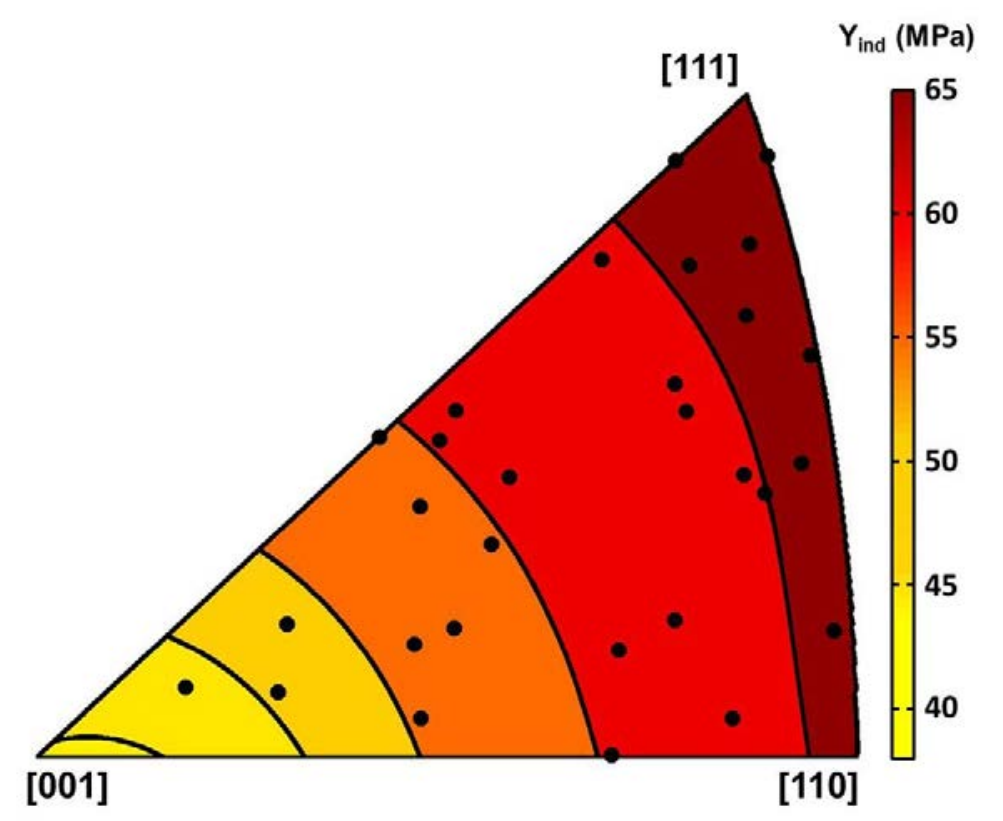

Figure 2: Contour plot generated by fitting a surface to the average $Y_{\text {ind }}$ values measured experimentally on annealed pure aluminum using spherical nanoindentation [32]. The black circles represent the orientations of the 27 grains tested. These values are used to establish the baseline values from which increases in $Y_{\text {ind }}$, as a result of the imposed plastic deformation, are measured.

The combined indentation-OIM analysis protocols developed in our prior work [32, 47] were based on the assumption that the effect of the crystal orientation on the measured indentation yield point can be expressed by the same multiplicative factor (very similar to a Taylor factor) for both undeformed and deformed samples. As argued in our previous work [32], this is a reasonable assumption for the high symmetry cubic crystals with a relatively large number of slips systems with roughly the same value of the slip resistance. The protocols start with establishing the dependence of the $Y_{\text {ind }}$ on the local crystal lattice orientation at the 
indentation site in the annealed sample, as this serves as the baseline (control) for subsequent quantification of the increase in the $Y_{\text {ind }}$ as a result of the imposed plastic deformation. This task was already experimentally accomplished for high purity aluminum [32]. This plot is reproduced in Figure 2, and is used here to account for the effect of local crystal orientation on $Y_{\text {ind }}$, since the same material was used in the present study.

For each of the indentations performed in the deformed condition, the expected $Y_{\text {ind }}$ in the fully annealed condition was extracted from Figure 2. In other words, this is the indentation yield strength that would have been measured for the selected orientation, if tested in the fully annealed condition. A reliable measure of the increase in $Y_{\text {ind }}$ (i.e., $\Delta Y_{\text {ind }}$ ) as a consequence of the macroscopically imposed plastic deformation (i.e., 20\% reduction by plane strain compression) can then be determined as the difference between the measured indentation yield point in the deformed condition (denoted as $Y_{\text {ind }}(g, d)$ ) and the estimated indentation yield point in the fully annealed condition (denoted as $Y_{\text {ind }}(g, 0)$ ) as

$$
\Delta Y_{\text {ind }}(g, d)=Y_{\text {ind }}(g, d)-Y_{\text {ind }}(g, 0)
$$

In Eq. (4) $d$ refers to the amount of macroscopically imposed plastic deformation and $g$ refers to the local crystal orientation at the indentation site.

The simplest relationship that one can try to establish between $Y_{\text {ind }}$ and the local dislocation content $(\rho)$ is through the critical resolved shear strength $\left(\tau_{c r s s}\right)$ of the slip system. This relationship can be expressed as

$$
\begin{aligned}
& Y_{\text {ind }}(g, d)=M(g) \tau_{\text {crss }}(d) \\
& \Delta \tau_{\text {crss }}(d)=\left(\tau_{\text {crss }}(d)-\tau_{\text {crss }}(0)\right) \propto \sqrt{\rho}
\end{aligned}
$$

where $M$ is similar to a Taylor factor for indentation that depends only on the grain orientation with respect to the indentation direction, $\tau_{\text {crss }}$ is the critical resolved shear strength in the crystal, $\Delta \tau_{c r s s}$ is the increase in the (averaged) critical resolved shear strength between the fully annealed and deformed conditions, and $\rho$ is the local dislocation density. It is further noted that only two of the three Bunge-Euler angles describing the local crystal orientation influence the measured 
value of $Y_{\text {ind }}$ (because an in-plane rotation of the sample in the spherical indentation experiment will not affect this measured value, even though the resulting stress and strain fields in the indentation zone are not axi-symmetric).

A convenient measure for comparing the relative hardening levels in the differently oriented regions in a given deformed sample is to express the increase in the slip resistance as a percentage of the initial critical resolved shear stress in the material in the annealed condition. Combining Eqs. (4)-(6), the percentage increase in the $\tau_{\text {crss }}$ is expressed as

$$
\% \tau_{\text {crss }}=\frac{\Delta \tau_{\text {crss }}(d)}{\tau_{\text {crss }}(0)} * 100=\frac{\Delta Y_{\text {ind }}(g, d)}{Y_{\text {ind }}(g, 0)} * 100
$$

As noted earlier, $\% \tau_{\text {crss }}$ provides an indirect measure of the local hardening (reflecting increase in local dislocation content) in the deformed samples (Eq. (6)).

The $\% \tau_{\text {crss }}$ values are expected to correlate to the Taylor factors associated with the macroscopically imposed plastic deformation on the sample. The Taylor factor $M$ is defined as

$$
M=\frac{\sum \Delta \gamma}{\Delta \varepsilon}
$$

where $\Delta \gamma$ is the sum of the slip shears on all the slip systems, and $\Delta \varepsilon$ is the imposed macroscopic plastic strain increment. In other words, a grain with a higher value of $M$ is expected to require a higher amount of total slip on its available slip systems to accommodate the imposed macroscopic plastic deformation. Grains with higher values of $M$ are generally referred as hard (oriented) grains, and those with lower values of $M$ are generally referred as soft grains. In our most recent work [32], we provided strong experimental evidence suggesting that grains with higher values of $M$ (i.e., hard grains) harden more than the grains with lower values of $M$ because they demand higher amounts of total slip in the grain. This previous study focused on grain interior regions. In this paper, we explore the grain boundary regions with the exact same toolsets. It is reiterated that $M$ discussed here is the Taylor factor corresponding to the plane strain compression experiment, and is not to be confused with the Taylor-like factor for indentation introduced in Eq. 5. Although, they have very similar physical interpretation, the two 
different $M$ values correspond to very different deformation modes, namely plane strain compression and indentation.

\section{Results and Discussion}

The eight grain boundaries studied here are identified on the OIM map of the 20\% deformed high purity aluminum sample shown in Figure 3. Each grain in the OIM map is color coded to represent its position on the standard [ND] inverse pole figure (IPF). In other words, the color represents the crystal direction in the grain that was parallel to the loading direction during the plane strain compression. With respect to the OIM map presented in Figure 3, the sample was allowed to expand freely in the horizontal direction (labeled as RD) and compressed along the vertical direction (labeled as ND). The out-of-the-paper direction is the constrained direction (labelled as TD) during the plane strain compression experiment. Note that the sample surface that was used for the nanoindentation testing is the TD section.

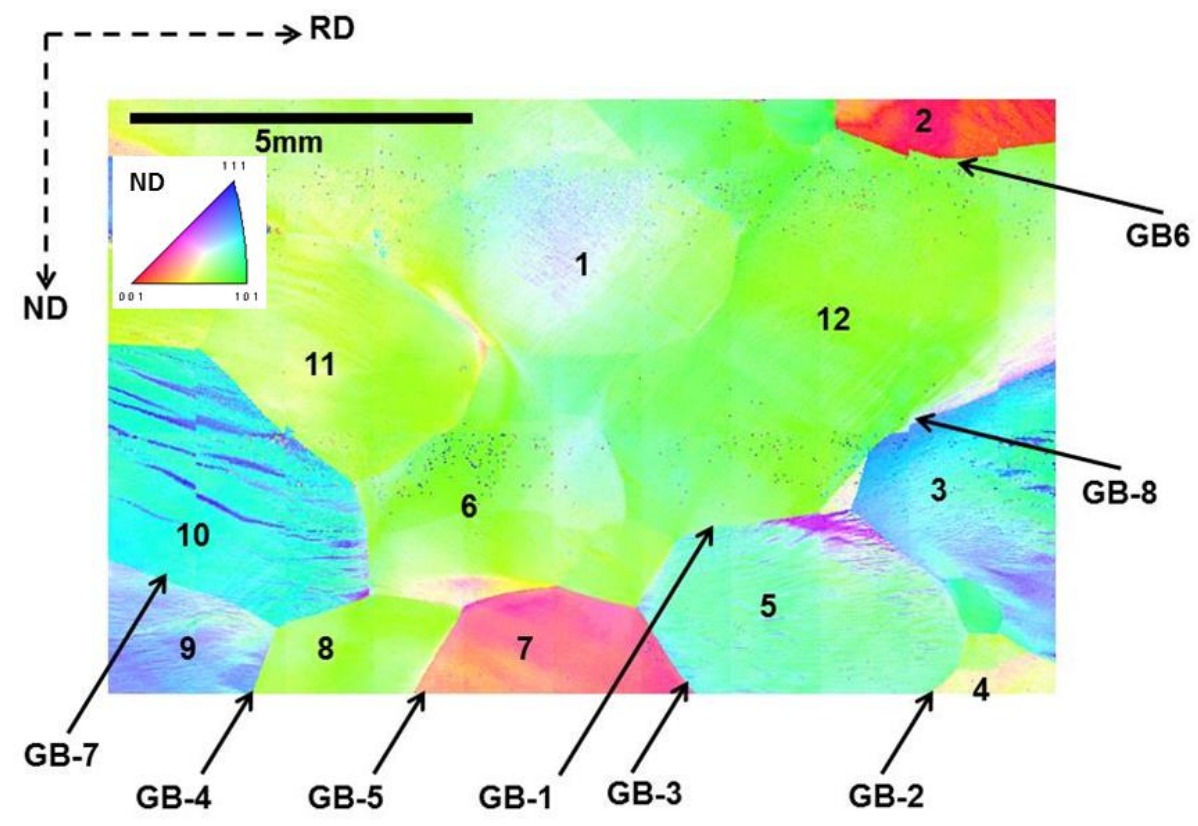

Figure 3: OIM map of the sample deformed to $20 \%$ reduction in height by plane strain compression. The specific grain boundaries studied using nanoindentation are marked. The discontinuities seen in grain 10 and grain boundaries 6 (GB-6) and 8 (GB-8) are artifacts of stitching several smaller OIM maps together. Note that ND was the loading direction during the plane strain compression in the channel die and the indentation tests were performed on the TD surface. 
About 60-100 indentation tests across each of the eight boundaries were carried out and the raw indentation data was converted into ISS curves. Representative ISS curves for indents close to and far away from the boundary, for the two grains (Grain \#5 and \#12) on either side of grain boundary \#1 (GB \#1) are shown in Figure 4. As discussed previously in Section 3, pop-ins are mostly absent in the indentation tests carried out on the deformed samples using the $20 \mu \mathrm{m}$ spherical tip, suggesting that it is relatively easy to set up the necessary dislocation sources in the indentation zones using the existing network of forest dislocations. Also clear from this figure is the fact that in the immediate vicinity of the boundary, the grains on either side of the boundary behave very differently. As seen in Figure 4, for grain \#5, there is little difference between the indentation response measured close to the grain boundary and away, whereas on the other side of GB \#1, in grain \#12, the measured ISS curve and the $Y_{\text {ind }}$ exhibit significant increases in the immediate neighborhood of the grain boundary than far away.
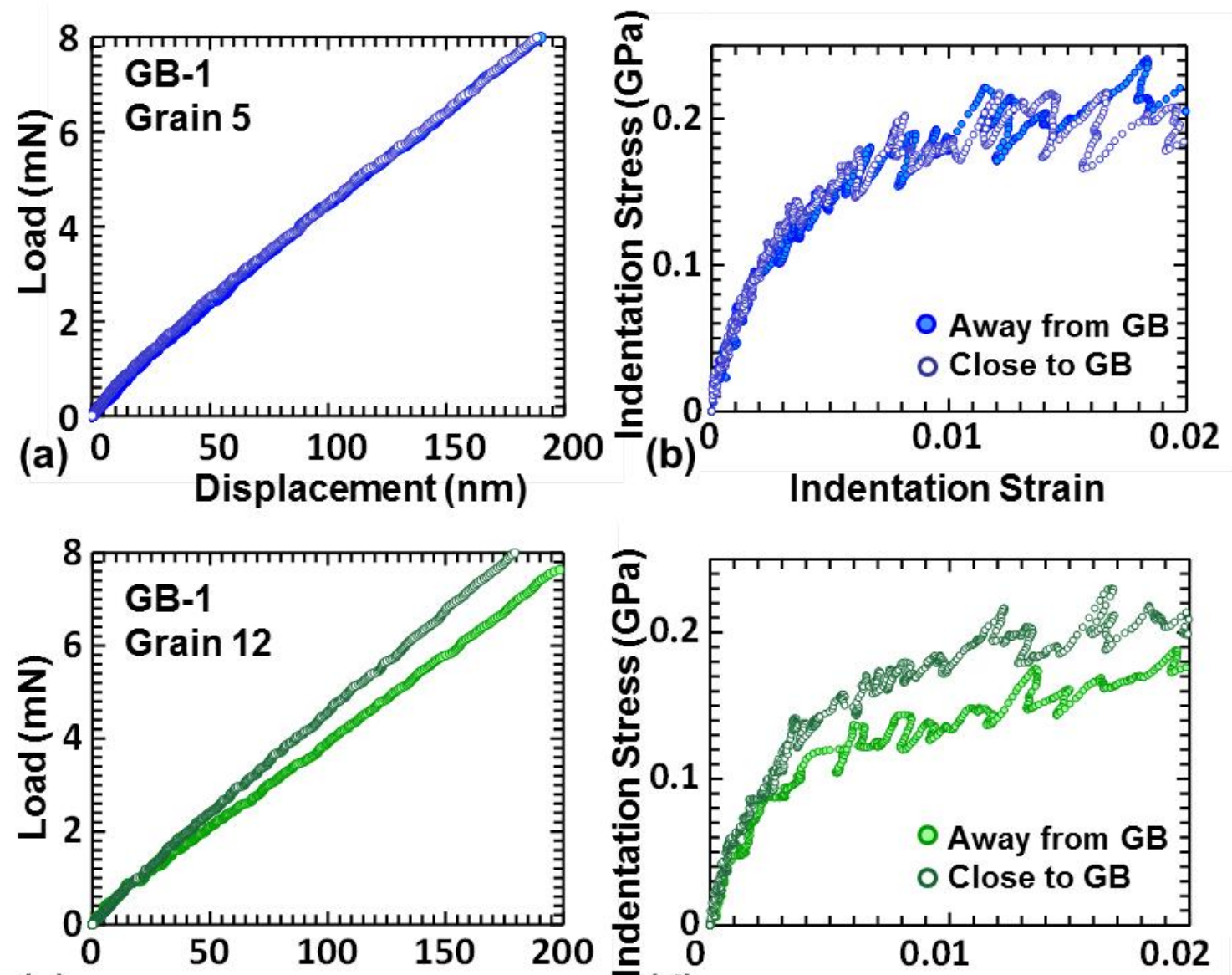

(c) Displacement $(\mathrm{nm})$

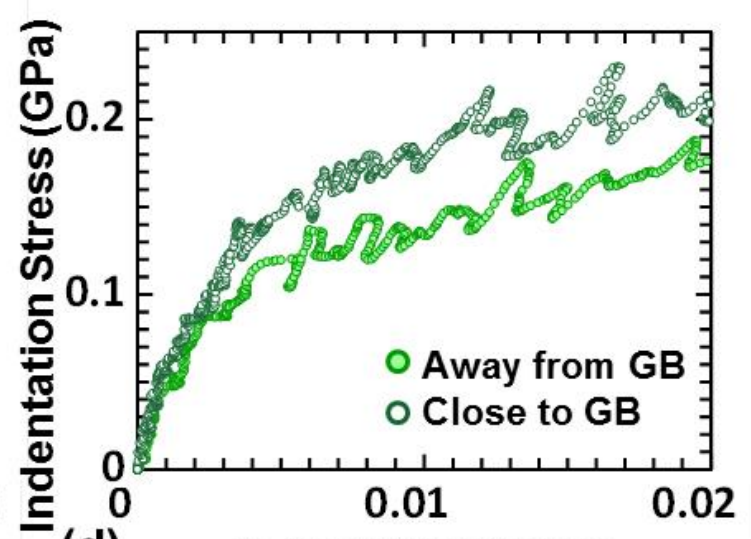

(d) Indentation Strain 
Figure 4: (a) and (c) Representative load-displacement curves. (b) and (d) Corresponding indentation stress-strain curves obtained for grains \#5 and \#12, respectively, close to $(\sim 10 \mu \mathrm{m})$ and away from $(\sim 40-50 \mu \mathrm{m})$ grain boundary 1 (GB-1).

The indentation test results for five of the eight boundaries studied are presented in Figures 5-9; these five represent the most interesting results from the total set of eight and communicate the overall trends observed in this work most effectively. The OIM map showing the grain boundary, the orientation on a standard [ND] inverse pole figure map and the position of the indents with respect to the grain boundaries are provided for each grain boundary in Figures 5-9 (a). The summaries of the measured $Y_{\text {ind }}$ and the percentage increase in $\tau_{\text {crss }}$ due to the imposed macroscopic deformation are given in Figures 5-9 (b) and (c) respectively. 

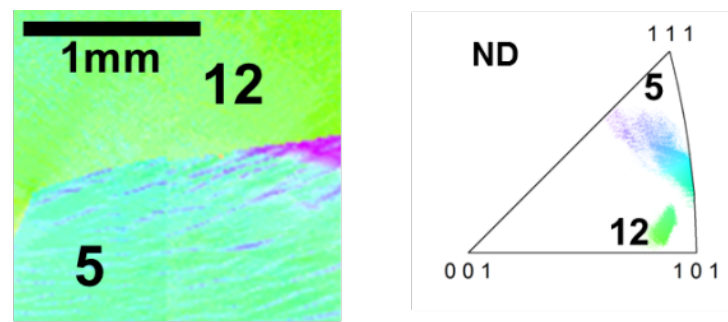

(a)
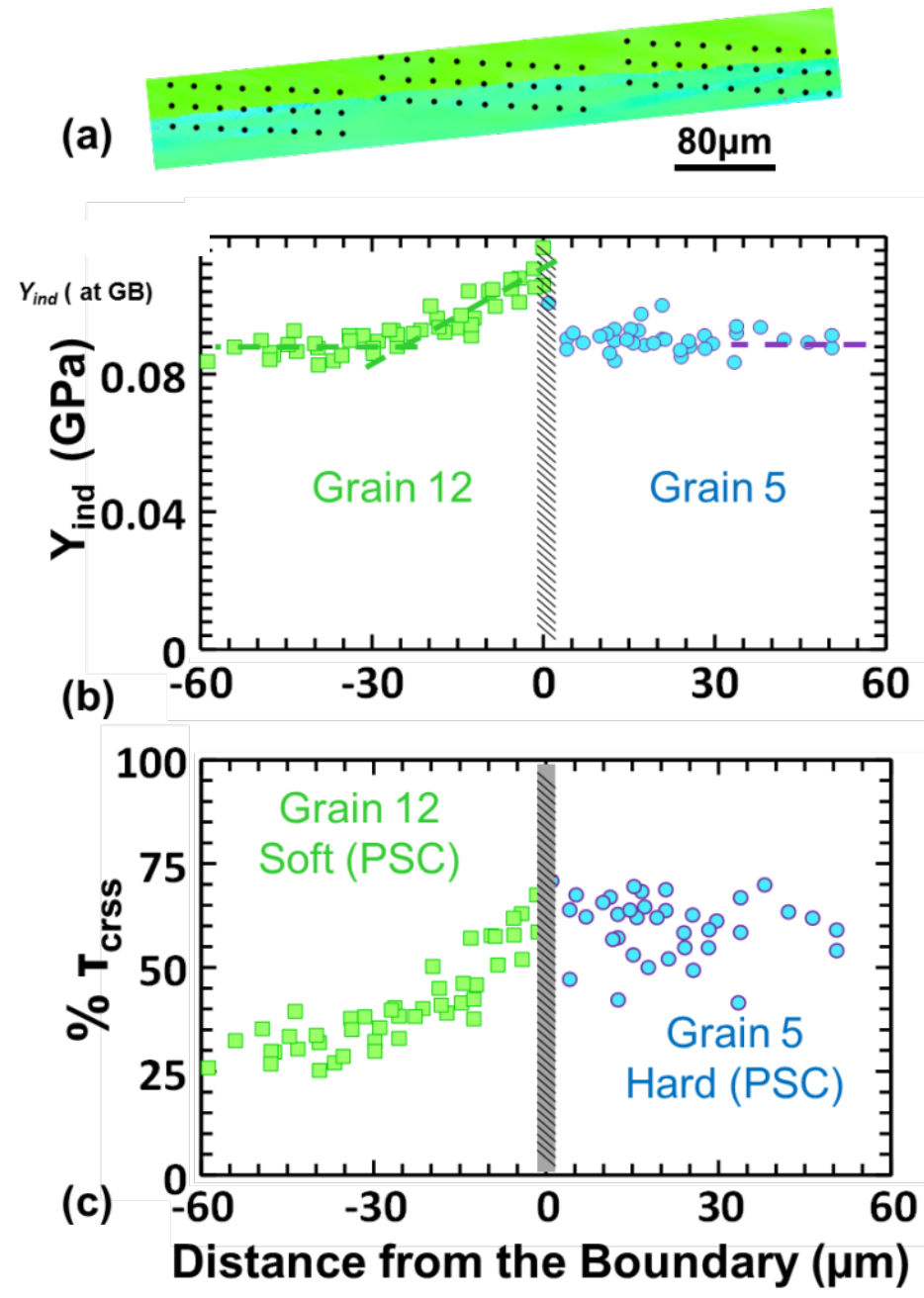

Figure 5: (a) Crystallographic details of grain boundary 1 (GB-1) and the location of the indents with respect to the boundary. (b) The measured indentation yield $\left(Y_{\text {ind }}\right)$ near GB1 between Grains \#12 and \#5. (c) Percentage change in $\tau_{\text {crss }}$ with respect to the annealed condition reflecting the local strain hardening. 


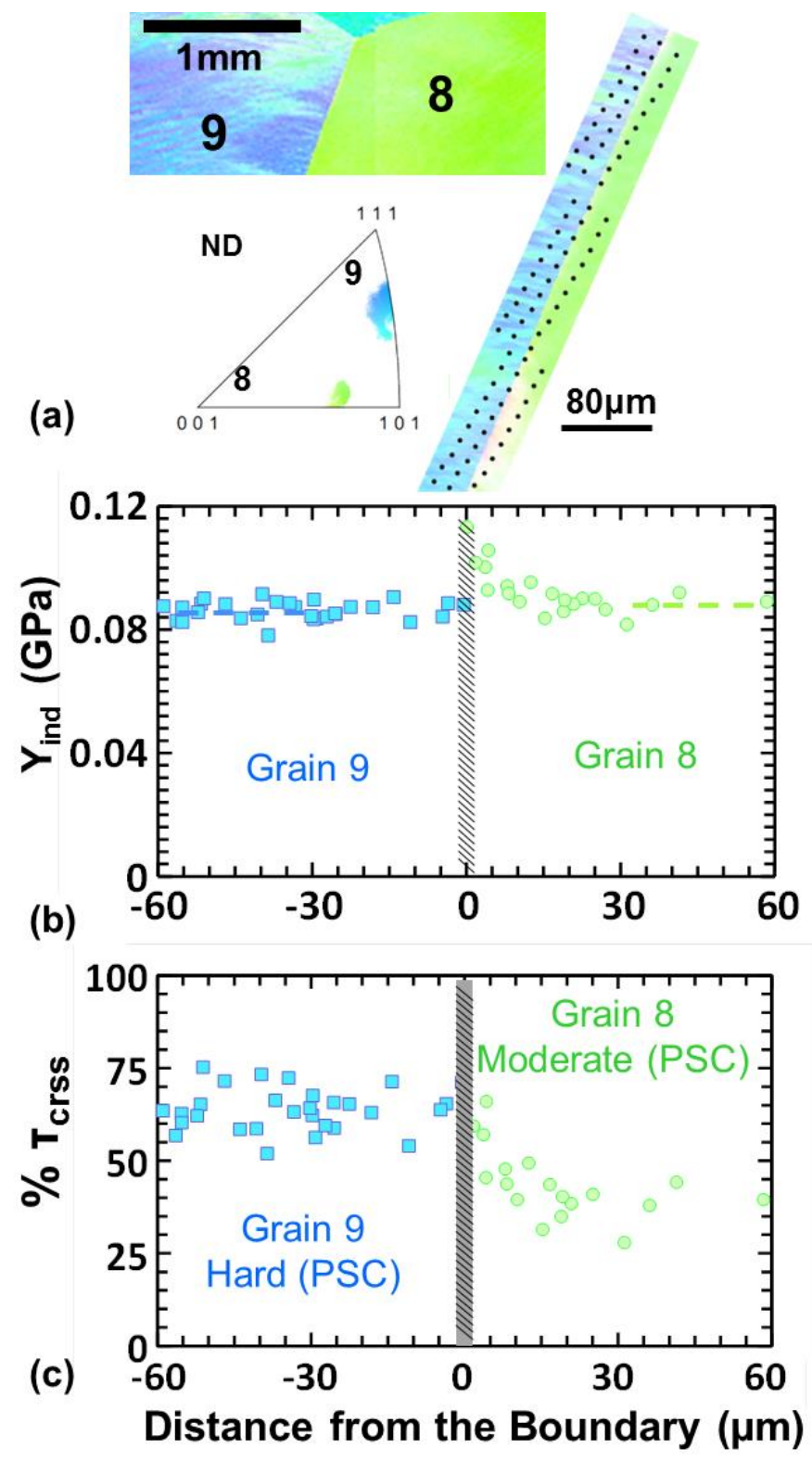

Figure 6: (a) Crystallographic details of the grain boundary 4 (GB-4) and the location of the indents with respect to the boundary. (b) The measured indentation yield $\left(Y_{\text {ind }}\right)$ near GB-4 between grains \#9 and \#8. (c) Percentage change in the $\tau_{\mathrm{crss}}$, with respect to the annealed condition reflecting the local strain hardening. 

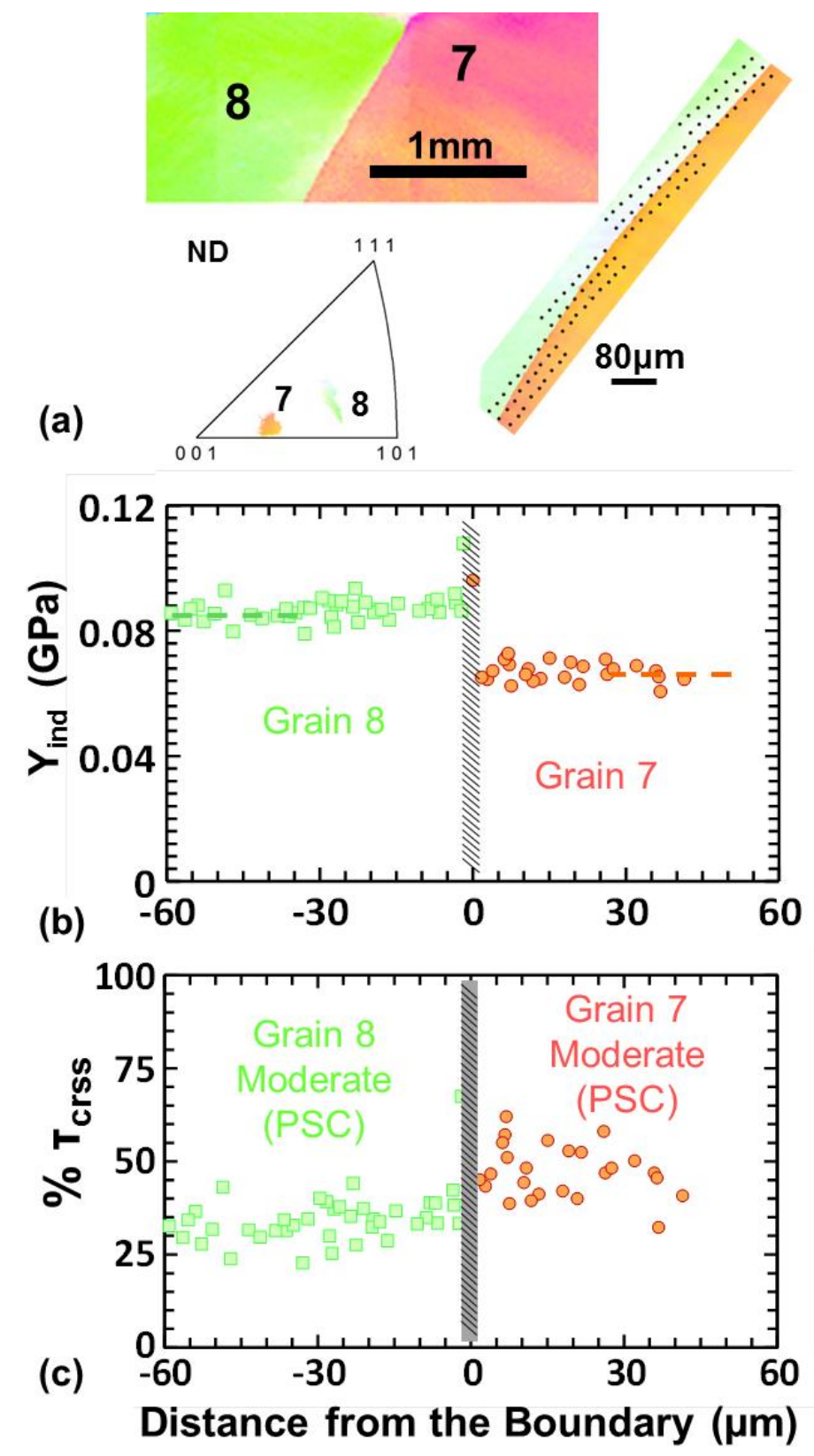

Figure 7: (a) Crystallographic details of the grain boundary 5 (GB-5) and the location of indents with respect to the boundary. (b) The measured indentation yield $\left(Y_{\text {ind }}\right)$ near GB5 between grains \#8 and \#7. (c) Percentage change in the $\tau_{c r s s}$, with respect to the annealed condition reflecting the local strain hardening.

Table 1 summarizes the details of the OIM and nanoindentation measurements away from the grain boundaries for all the eight boundaries of interest. The orientation 
away from the grain boundary represents the average orientation as far as $500 \mu \mathrm{m}$ from the region of the grain boundary where the GB tests were performed. $\Delta \mathrm{g}$ across the boundary is the calculated misorientation between the orientations away from the grain boundary, measured as described above. The Taylor factors presented are for plane strain compression, and are calculated using the average orientations measured away from the boundary, as reported in this table. $Y_{\text {ind }}(g, d)$ and the estimated $Y_{\text {ind }}(g, 0)$ represent the indentation yield strength values measured within the grains ( $500 \mu \mathrm{m}$ from the grain boundaries) and the indentation yield strength values for the average orientations measured away from the grain boundaries, extracted from the indentation yield surface plot for fully annealed aluminum shown in Figure 2.

Table 2 summaries the details of the OIM and nanoindentation measurements in the vicinity of the grain boundaries, for the eight boundaries of interest. The orientation at the grain boundary represents the average orientation in the region up to $60 \mu \mathrm{m}$ away from the grain boundary, over the distance along the boundary where the indentation tests were performed. $\Delta \mathrm{g}$ across the boundary is the misorientation between the average orientations measured on either side of the grain boundary, as described above. The Taylor factors (at GB) presented are for plane strain compression for the average orientations in the grain boundary region. $Y_{\text {ind }}(g, 0)$ represents the indentation yield strength values for the orientations measured in the grain boundary region extracted from the indentation yield plot for fully annealed aluminum (Figure 2).

From Figures 5-9 and Tables 1 and 2, it is clear that not all grain boundaries behave in the same manner. For GB-1 (See Figure 5), there is a substantial transition zone on one side of the boundary where the measured values of $Y_{\text {ind }}$ near the boundary are higher than those measured within the grains (away from the boundary region). The immediate vicinities of GB-2, GB-3 and GB-4 (see Figure 6), also exhibit hardened layers, at least on one side of the grain boundary. For GB-5 (see Figure 7) and GB-6, the presence of a grain boundary appears to have a negligible effect on the $Y_{\text {ind }}$ values measured in the vicinity of the grain boundary. In other words, the $Y_{\text {ind }}$ measured very close to the grain boundary is very similar to the $Y_{\text {ind }}$ measured well away from the boundary for grains on both sides of this boundary. Note that all these boundaries GB-1 to GB-6 were high angle grain boundaries. GB-7 is a low angle grain boundary, and once again, the presence of a grain boundary appears to have a negligible effect on the $Y_{\text {ind }}$ values measured in the vicinity of the grain boundary (see Figure 8). Also, note that there seems to be no 
correlation between the presence of a hardened layer at the grain boundary regions and the grainto-grain misorientation angle across the grain boundaries. This is a very important observation as it is generally believed that the grain-to-grain misorientation plays a significant role in determining the local material response, near the grain boundary, during plastic deformation. Clearly, additional studies involving many more grain boundaries are needed to confirm this finding.

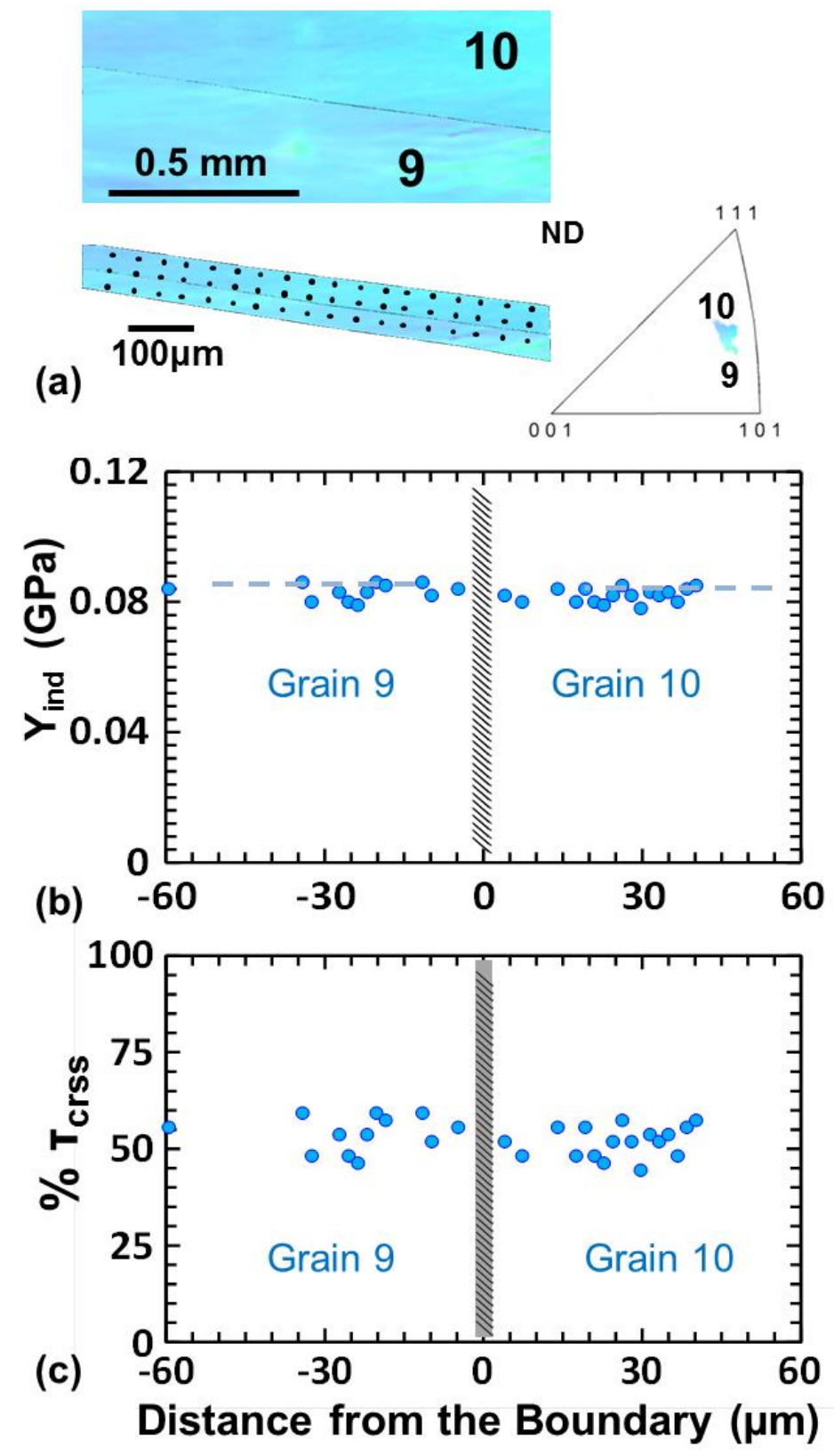


Figure 8: (a) Crystallographic details of the grain boundary 7 (GB-7) and the location of indents with respect to the boundary. (b) The measured indentation yield $\left(Y_{\text {ind }}\right)$ near GB-7 between grains \#9 and \#10. (c) Percentage change in the $\tau_{\text {crss }}$, with respect to the annealed condition reflecting the local strain hardening. Note that this is a low (grainto-grain misorientation) angle grain boundary (All other boundaries studies are high (grainto-grain misorientation) angle boundaries).

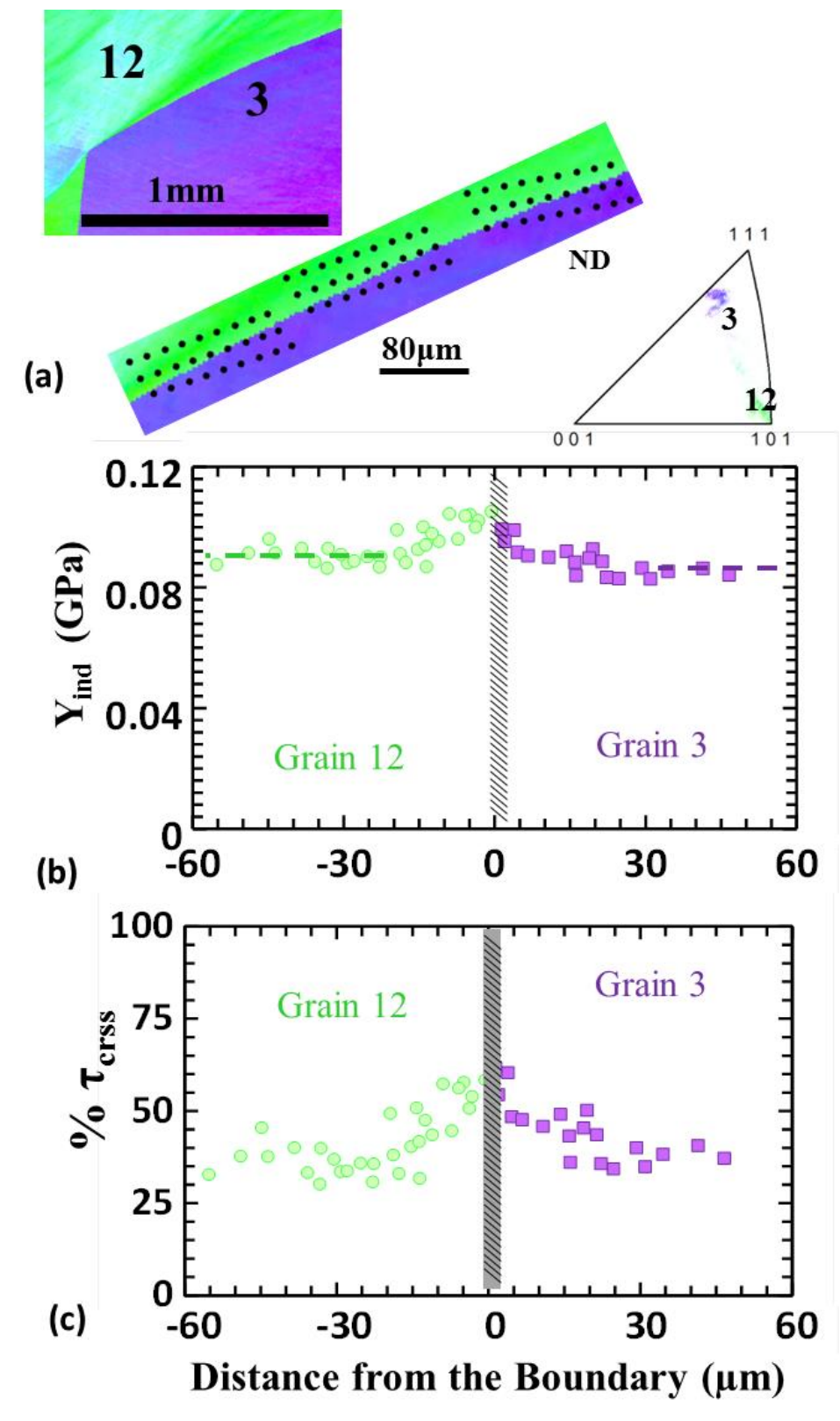


Figure 9: (a) Crystallographic details of the grain boundary 8 (GB-8) and the location of indents with respect to the boundary. (b) The measured indentation yield ( $\left.Y_{\text {ind }}\right)$ across GB-8 between grains \#12 and \#3. (c) Percentage change in the $\tau_{\text {crss, }}$, with respect to the annealed condition reflecting the local strain hardening.

\begin{tabular}{|c|c|c|c|c|c|c|c|c|c|c|c|c|c|c|c|c|c|}
\hline 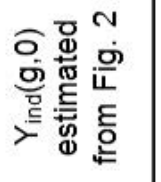 & $\frac{\pi}{0}$ & กิ & $\mathscr{0}$ & $\widetilde{\widetilde{C}}$ & 요 & 요 & 守 & กิ & $\widetilde{6}$ & $\mathscr{q}$ & $\mathscr{0}$ & ภூ & 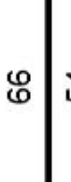 & 茴 & ஜீ & $\bar{\emptyset}$ & $\mathscr{8}$ \\
\hline 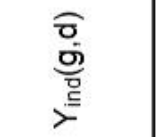 & $\stackrel{0}{0}$ & ৪ & ৪। & $\underset{\infty}{\infty}$ & $\widehat{\infty}$ & $\begin{array}{l}\infty \\
\infty\end{array}$ & $\begin{array}{l}\infty \\
0 \\
0\end{array}$ & R & Б̄ & @ & $\begin{array}{l}\infty \\
\infty\end{array}$ & 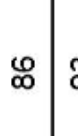 & ด̆ & 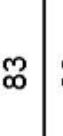 & న్ల & $\hat{\infty}$ & б̄ \\
\hline $\begin{array}{l}\text { D } \\
\text { O }\end{array}$ & ฮ & $\begin{array}{l}\hat{b} \\
\mathrm{~m}\end{array}$ & $\mid \begin{array}{c}\mathbb{T} \\
-6 \\
-\end{array}$ & $\frac{ \pm}{i}$ & $\frac{\infty}{i}$ & 足 & | & $\begin{array}{l}\mathrm{H} \\
\mathrm{N} \\
\mathrm{i}\end{array}$ & \begin{tabular}{l}
$\infty$ \\
\multirow{r}{\sim}{} \\
$i$
\end{tabular} & $\stackrel{?}{\stackrel{R}{r}}$ & $\frac{ \pm}{i}$ & $\stackrel{\text { ๑ }}{\leftarrow}$ & 苾 & $\begin{array}{l}\bar{\infty} \\
0 \\
0\end{array}$ & \begin{tabular}{c}
$\infty$ \\
$\infty$ \\
\hdashline \\
0
\end{tabular} & 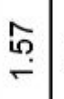 & $\bar{\infty}$ \\
\hline 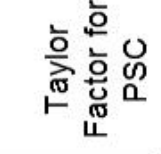 & & $\begin{array}{l}\hat{f} \\
\dot{v}\end{array}$ & 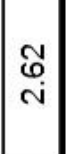 & $\begin{array}{l}\mathcal{N} \\
\dot{m} \\
\end{array}$ & $\begin{array}{l}\mathscr{0} \\
\mathscr{\sigma} \\
\dot{\nabla}\end{array}$ & 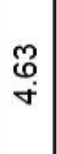 & $\begin{array}{l}\infty \\
\stackrel{m}{m} \\
m\end{array}$ & $\frac{\mathfrak{N}}{\check{r}}$ & $\underset{\sim}{\stackrel{\nabla}{*}}$ & $\underset{m}{\stackrel{m}{m}}$ & $\underset{丶}{\check{m}}$ & $\stackrel{\hat{m}}{\mathrm{i}}$ & $\stackrel{d}{N}$ & $\begin{array}{l}\text { P्ల } \\
\forall \\
\forall\end{array}$ & $\underset{\sim}{\bar{*}}$ & $\begin{array}{l}\stackrel{8}{0} \\
\dot{m}\end{array}$ & $\begin{array}{l}\infty \\
\infty \\
\end{array}$ \\
\hline 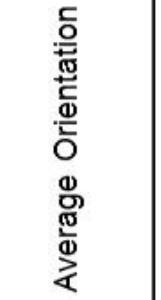 & $\begin{array}{l}\tilde{\theta} \\
\dot{\theta} \\
\dot{\theta}\end{array}$ & 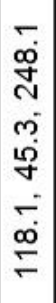 & 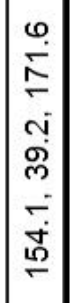 & 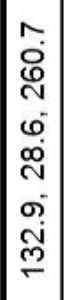 & 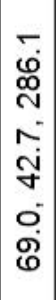 & 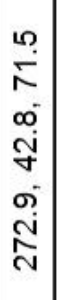 & 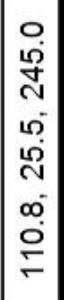 & 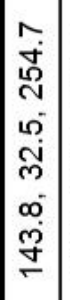 & 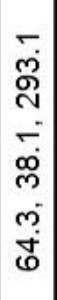 & 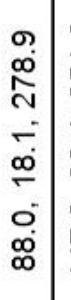 & 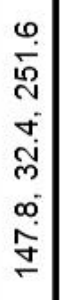 & 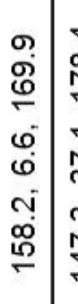 & 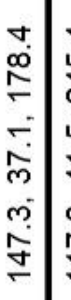 & 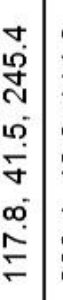 & 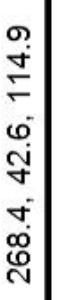 & 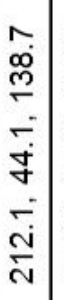 & 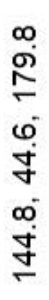 \\
\hline $\begin{array}{l}\frac{0}{2} \\
\frac{1}{\sqrt[1]{10}} \\
\text { 0 }\end{array}$ & & 10 & 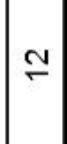 & $\nabla$ & 10 & $\infty$ & $\wedge$ & の & $\infty$ & $\sim$ & $\infty$ & $N$ & $\cong$ & の & 으 & $m$ & $\stackrel{N}{ }$ \\
\hline 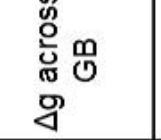 & ฮ & & Vy & F & & & ? & है & & ஓं & $\dot{b}$ & 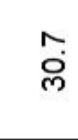 & & $\overline{\mathrm{N}}$ & & $\begin{array}{r}\text { vi } \\
\text { v }\end{array}$ & \\
\hline 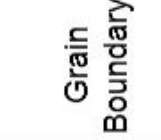 & & & m & $\begin{array}{l}\text { mे } \\
\text { co }\end{array}$ & & C & ض̃ & $\begin{array}{l}y \\
0 \\
0\end{array}$ & & 息 & b́ & $\begin{array}{l}\varphi \\
\text { ஸे } \\
\text { On }\end{array}$ & & 灾 & & 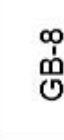 & \\
\hline
\end{tabular}

Table 1: Summary of OIM and nanoindentation measurements up to $500 \mu \mathrm{m}$ away from the grain boundaries, for the 8 grain boundaries studied in this work. 


\begin{tabular}{|c|c|c|c|c|c|c|c|c|c|c|c|c|c|c|c|c|c|}
\hline 잉 & & $\stackrel{\circ}{\circ}$ & $\stackrel{N}{N}$ & 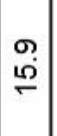 & $\stackrel{\circ}{\circ}$ & $\begin{array}{l}\circ \\
0\end{array}$ & $\begin{array}{l}\stackrel{+}{ \pm} \\
\pm\end{array}$ & $\begin{array}{l}\sigma \\
\infty\end{array}$ & $\stackrel{0}{=}$ & 웅 & $\stackrel{\circ}{\circ}$ & $\begin{array}{l}0 \\
0\end{array}$ & 웅 & $\stackrel{\circ}{\circ}$ & $\stackrel{\circ}{\circ}$ & $\stackrel{10}{\check{C}}$ & ம் \\
\hline 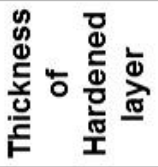 & 豆 & 0 & $\stackrel{\infty}{\sim}$ & 志 & 0 & 0 & $\approx$ & 0 & $\sigma$ & 0 & 0 & 0 & 0 & 0 & 0 & 유 & $\underset{\leftarrow}{\longleftarrow}$ \\
\hline 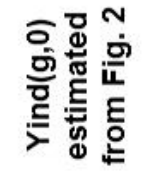 & $\frac{\pi}{0}$ & 饮 & $\mathscr{\bullet}$ & $\widetilde{\mathscr{O}}$ & 守 & 5 & 윰 & กิ & ષ゙ & \% & Ðே & $\bar{\sigma}$ & $\mathscr{O}$ & டீ & $\mathscr{\bullet}$ & $\widetilde{\mathscr{\sigma}}$ & $\mathscr{0}$ \\
\hline 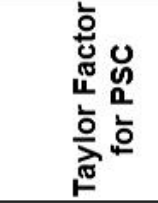 & & $\begin{array}{l}\stackrel{0}{0} \\
\stackrel{0}{*}\end{array}$ & $\begin{array}{l}0 \\
\infty \\
\text { Ni }\end{array}$ & $\begin{array}{l}\infty \\
\stackrel{\infty}{ } \\
\text { ஸे }\end{array}$ & $\begin{array}{l}\overleftarrow{\sigma} \\
\dot{\sigma}\end{array}$ & $\begin{array}{l}\hat{6} \\
\dot{\sigma}\end{array}$ & $\begin{array}{l}\infty \\
m \\
m \\
m\end{array}$ & $\underset{丶}{\check{m}}$ & 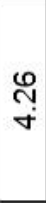 & $\begin{array}{c}\hat{m} \\
\text { m. }\end{array}$ & 으. & $\begin{array}{l}\text { m } \\
\text { i }\end{array}$ & $\begin{array}{l}\stackrel{0}{0} \\
\text { N }\end{array}$ & $\begin{array}{l}\tilde{m} \\
\stackrel{\nabla}{*}\end{array}$ & $\underset{⿱}{\stackrel{J}{*}}$ & 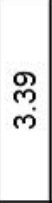 & $\check{\check{m}}$ \\
\hline 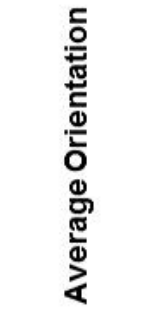 & $\begin{array}{l}\tilde{\sigma} \\
\dot{\theta} \\
\bar{\sigma}\end{array}$ & 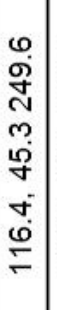 & 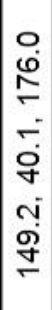 & 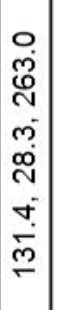 & 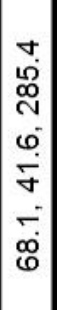 & 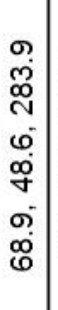 & 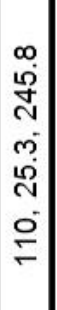 & 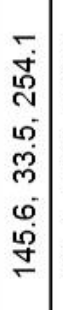 & 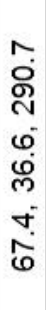 & 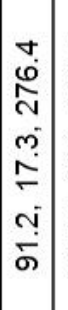 & 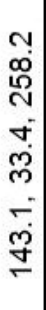 & 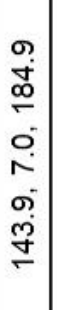 & 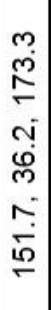 & 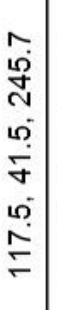 & 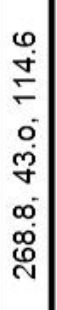 & 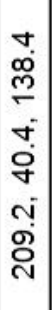 & 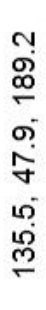 \\
\hline $\begin{array}{l}\text { 은 } \\
\text { 든 } \\
\text { 인 }\end{array}$ & & 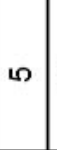 & $\cong$ & $\nabla$ & م & $\infty$ & $\Lambda$ & の & $\infty$ & $N$ & $\infty$ & $N$ & $\cong$ & の & 으 & $m$ & $\stackrel{\sim}{\longleftarrow}$ \\
\hline 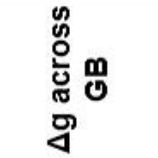 & $\widetilde{0}$ & 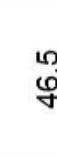 & : & $\stackrel{m}{\nabla}$ & & m & & กิ & & ர্ল & & $\begin{array}{l}\mathscr{1} \\
\text { ภ }\end{array}$ & & $\stackrel{\infty}{\digamma}$ & & & \\
\hline 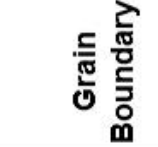 & & 宅 & '́ & 宪 & & 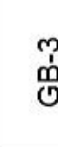 & & $\begin{array}{l}\text { '. } \\
\text { هُ }\end{array}$ & & 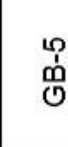 & & 崩 & & 怘 & & & \\
\hline
\end{tabular}

Table 2: Summary of OIM and nanoindentation measurements near $(-60 \mu \mathrm{m})$ the grain boundary region, for the 8 grain boundaries studied in this work.

Looking at the Taylor factors in plane strain compression for each of the orientation of interest, it is seen that away from the grain boundaries, the orientations with a higher Taylor factor show more hardening as a result of the $20 \%$ height reduction. This is consistent with the findings reported previously [32]. 
However, in the immediate neighborhood of the grain boundary, a grain with a low Taylor factor (soft grain) when present next to a grain with relatively higher Taylor factor (hard grain) showed much more hardening at the boundary. The difference in the Taylor factor between adjacent grains is highest across GB-1, where grain \#12 is a very soft grain with a Taylor factor of 2.62 next to a very hard grain (Taylor factor of 4.47). From Figure 5 and Tables 1 and 2, it is seen that grain \#12, in the immediate vicinity of GB-1 shows the most significant amount of hardening both in terms of the percentage increase in the $Y_{\text {ind }}$ (with respect to the grain interior) as well as the thickness of the hardened layer. GB-2, GB-3 and GB-4 show progressively lesser difference in the Taylor factors for the orientations on either side of the boundary and also progressively lesser amount of hardening on the 'soft grain' side. GB-5 has two moderately hard grains, whose Taylor factors are very similar, on either side of the boundary. GB-6 has two soft grains of very similar Taylor factors next to each other. In both these cases, the hardened layer is completely absent. GB-7 is a low angle grain boundary with two relatively hard grains next to one another across the boundary. Consistent with previous observations (GB-5 and GB-6), here again, there is little effect of the boundary on the hardening near the grain boundary. Note that all low angle grain boundaries will have grains of very similar orientation and Taylor factors next to each other.

While the seven of the grain boundaries studied (GB-1 through GB-7) followed the trends discussed above, GB-8 appears to be an exception. GB-8 has a soft grain \#12 (Taylor factor of 2.88) adjacent to a moderately hard grain \#3, that has a Taylor factor of 3.60. According to previous observations, one would expect a hardened layer on the side of grain \#12. However in this case, as seen in Figure 9, there is a significant hardened layer present on either side of the grain boundary. In this study, only the orientations after the macroscopic deformation is available and information about the starting orientation (before deformation) and the path followed during deformation is missing. Grain \#3 is a large grain at the free surface of the sample, and therefore its response can be substantially different from that of the other grains in the sample. This can also be the reason for the high degree of fragmentation within grain \#3 as compared to the rest of the grains (see Figure 3). Since grain \#3 is one of the grains adjoining GB-8, it could be a factor in the response observed at this grain boundary. Additional studies are clearly needed to document the time evolution of the microstructure in the grain boundary regions to extract deeper insights. 


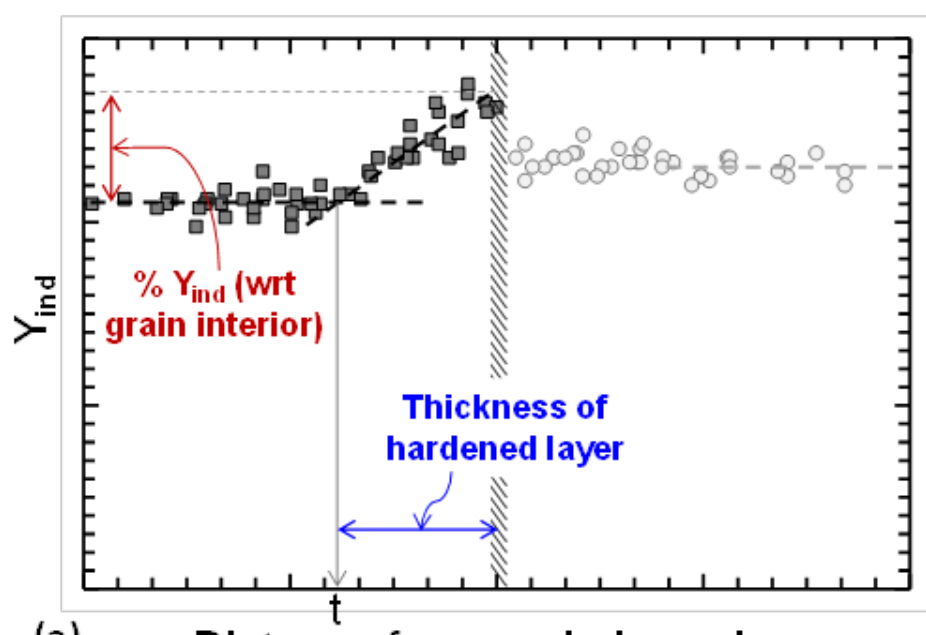

(a) Distance from grain boundary

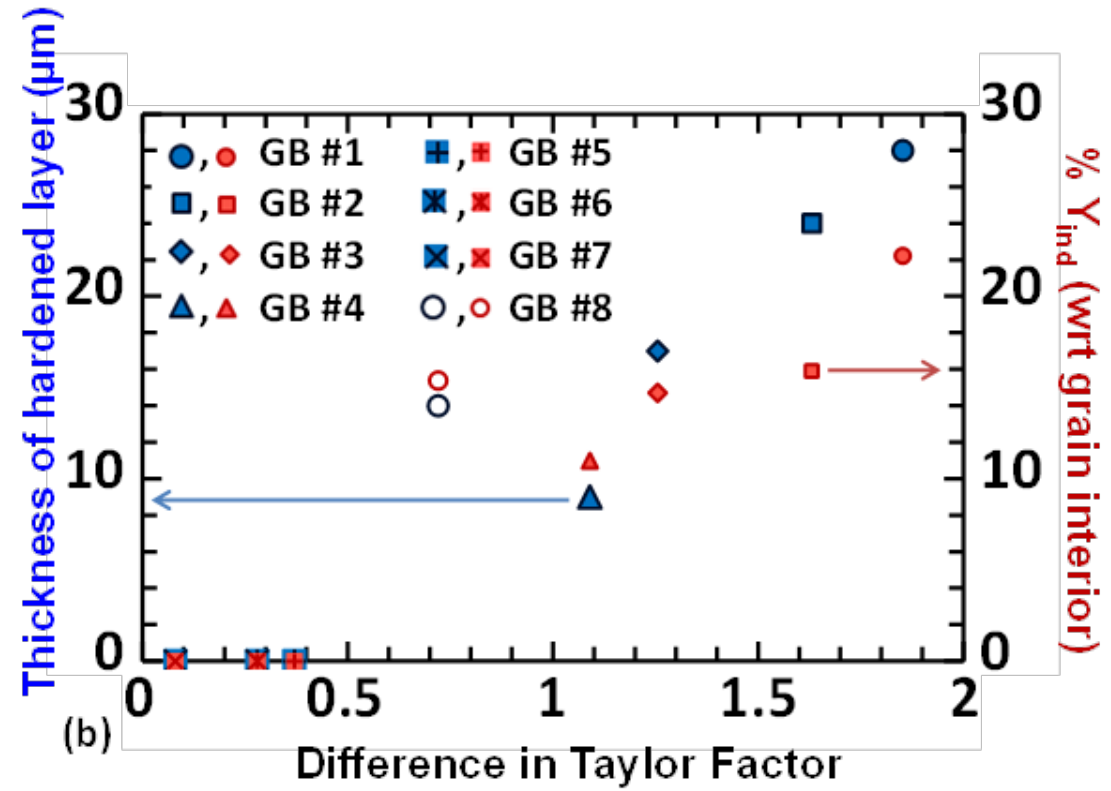

Figure 10: (a) Schematic showing the parameters used to describe the hardening levels in the grain boundary regions. (b) The variation of the hardening parameters, namely the thickness of the hardened layer and the percentage increase in the $Y_{\text {ind }}$ (with respect to the grain interior) for the 'soft' grain all the grain boundaries studied, as a function of the difference in the Taylor factor for the two grain across the boundary.

In order to quantify the strain hardening in the grain boundary regions, two parameters are defined. The first parameter is the thickness of hardened layer $(\mathrm{t})$ and the second parameter is the percentage change in the indentation yield $\left(\% \Delta Y_{\text {ind }}\right)$ measured at the grain boundary with 
respect to that in the grain interior ( $500 \mu \mathrm{m}$ away from the boundary). A schematic, defining the two parameters employed to describe the hardening at the grain boundaries studied in this work, is shown in Figure 10a. The last two columns in Table 2 provide details about the same two parameters for all the grain boundaries tested. Note here that the calculated $\% \Delta Y_{\text {ind }}$ and the measured thickness of the hardened layer are both strongly affected by the number of data points used for the interpolation and hence it is important to have a sufficiently large number of data points in the 'hardened region' in order to be confident about the numbers extracted.

Figure 10b shows the variation of the two grain boundary parameters (reported in Table 2) defined above as a function of the difference in the Taylor Factor (reported in Table 1) of the grains on either side of the grain boundary in question. Note that the grain boundary parameters are consistently estimated using the softer grain as the reference (in computing the $\% \Delta Y_{\text {ind }}$ ). There appears to be a very strong relationship between the difference in Taylor factor at the grain boundary and the extent of hardening at the grain boundary. Although more data points need to be added to this plot in order to reliably quantify the relationship, this study presents important evidence to substantiate prior hypotheses in literature about the complex interactions between grains [38, 59] and the presence of hardened layers at grain boundaries in deformed materials [4, 60]. More specifically, the measurements reported in this work indicate that these hardened layers are predominantly on the side of the softer (lower Taylor factor) grain. Note that the grain boundary parameters for GB \#8 are plotted using a different symbol (open circles) because the response at this grain boundary was different from all other boundaries studied (i.e., hardening was observed on both sides of the boundary). Because of the small data size, it is very difficult to conclude if the observation at GB\#8 is substantially different from the other GBs or if it is within the range of expected variance in the measurements obtained in this study. Nevertheless, an important observation from Figure 10(b) is that the thickness of the hardened layer observed near the grain boundaries is comparable to the average grain sizes in most commercially used metals and alloys. Therefore, for most materials of interest in real applications, the grain boundaries between hard and soft grains will produce significant hardening within the entire soft grain [38].

Measures of the grain orientation spread (GOS) and grain average misorientation (GAM) were extracted from the OIM maps at different scales: (i) regions up to 500 $\mu \mathrm{m}$ away from the boundary, and (ii) regions up to $\sim 60 \mu \mathrm{m}$ from the grain boundaries. The GOS values ranged between $1^{\circ}$ and $4^{\circ}$, and the GAM values were found to be within the range of $0.3^{\circ}$ to $0.8^{\circ}$. While 
the GOS and GAM values were slightly higher for regions near the grain boundary as compared to those measured for regions up to $\sim 500 \mu \mathrm{m}$ away from the boundary, there were no strong correlations between the presence of a hardened layer at grain boundaries and the increase in the measured GOS or GAM. Although the higher GOS and GAM values may have an influential effect on any subsequent recrystallization, they did not appear to have a strong influence on the local strain hardening behavior studied here. This is probably because the grain misorientations measured in this study only capture the geometrically necessary component of the defect density at a fairly coarse length scale ( $1 \mu \mathrm{m}$ in our experiments). In reality, the local flow strength would be dependent on the entire defect density (including both geometrically necessary and the statistically stored components).

It should be noted that the grains in the samples studied were grown after the samples were cut from larger pieces. As the grains grow very large during the long annealing treatment (72 hours at $640^{\circ} \mathrm{C}$ ), the grain boundaries very close to the surfaces, will migrate very slowly and will have time to orient themselves at almost right angles to the free surface. Therefore we expect the grain boundaries we are investigating to be nominally perpendicular to the sample surface, particularly within the relatively shallow depths that we are interested in here. This was also verified by removing a top layer of about $100 \mu \mathrm{m}$ and re-imaging the sample surface using OIM. Thus, the possibility of a grain boundary being inclined to the sample surface is very remote. Another concern in the interpretation of the observed changes in local mechanical properties near the grain boundaries is that there might be artifacts resulting from curvatures on the sample surface at the grain boundary regions due to sample preparation, especially during the electropolishing step. In order to exclude this as a possibility, the topography of the sample surface around two grain boundaries of interest (GB-1 and GB-6) was imaged with a sharp Berkovich tip using Hysitron's TI 950 TriboIndenter ${ }^{\circledR}$ in the SPM imaging mode. For both these boundaries, it was observed that the sample surface was very smooth and that the maximum height difference at the grain boundary was $\sim 8 \mathrm{~nm}$, and occurred within a distance of $\sim 2 \mu \mathrm{m}$ around the boundary. Any data points affected by such steps (if present) at the boundary would lie within a region of $\pm 1 \mu \mathrm{m}$ from the grain boundary (grey-ed out on the plots in Figures 5-9 (b,c)). This region of $\pm 1 \mu \mathrm{m}$ from the boundary is also the region where the indentation zone at the point of yield might include the grain boundary, which makes the extraction of the 
indentation yield values from these tests difficult to interpret. Consequently, these measurements were excluded from the analyses and results presented in this paper.

The presence of a hardened layer in the vicinity of certain grain boundaries (when a hard grain was present next to a soft grain) observed in this work is distinctly different from a prior study in 30\% deformed, large grained Fe-3\%Si using a similar approach, reported by Pathak et al [33]. In the Fe-3\%Si, it was reported that for a high angle grain boundary, the transition zone of $\sim 30 \mu \mathrm{m}$ was present on the side of the hard grain and within this transition zone, a gradual decrease in the indentation yield strength $\left(Y_{\text {ind }}\right)$ was observed. In other words, the local dislocation density was lower in the immediate proximity of the boundary suggesting that the boundary in question acted as a dislocation sink (on the hard grain side). It should however be noted that Fe-3\%Si has a bcc structure while aluminum is fcc. However, since results for only one high angle boundary were reported for Fe-3\%Si, it is difficult to say if the all boundaries in Fe-3\%Si would show similar trends. In order to further explain these observations, one needs to understand dislocation-grain boundary interactions (including absorption, desorption and transmission mechanisms [14]). Clearly, additional investigations are needed to further confirm the trends and conclusions reported in both the earlier work and this work.

It is noted that our discussion here has been focused primarily on the strain hardening in the vicinity of grain boundaries. However, it is anticipated that the protocols developed and used in this study can be extended and employed to study a number of other phenomena related to grain boundary regions such as damage nucleation and growth [14], recrystallization [61], microcracking due to hydrogen embrittlement [62].

\section{Conclusions}

A novel approach to characterizing the mechanical behavior of regions close to grain boundaries in deformed polycrystalline materials, using combined OIM-spherical nanoindentation, is demonstrated in this study on aluminum polycrystals. From the regions studied around eight selected grain boundaries, it is concluded that Taylor 'soft' grains when present next to Taylor 'hard' grains harden significantly more in the immediate vicinity of the boundary. The extent of hardening increases with the increase in the difference in the Taylor factor for the adjacent grains. In other words, spatial variations in stored energy are significant in the vicinity of the boundary when a 'hard' grain is present adjacent to a 'soft' one. Interestingly, 
no correlation was observed either between the hardening at the grain boundary regions and grain-to-grain misorientation angle across the grain boundaries or between the hardened layer at grain boundaries and the increase in the measured grain orientation spread in the vicinity of the boundaries.

\section{Acknowledgement}

Authors acknowledge funding from U.S. Department of Energy, Office of Nuclear Engineering, Nuclear Engineering Enabling Technologies (2013 DOE-NEET). The MTS nanoindenter XP and the TSL-OIM system (integrated with the ESEM Philips XL-30) used in this study is maintained and operated by the Centralized Research Facilities in the College of Engineering at Drexel University. SJV is currently at Los Alamos National Laboratory. Los Alamos National Laboratory, an affirmative action equal opportunity employer, is operated by Los Alamos National Security, LLC, for the National Nuclear Security Administration of the U.S. Department of Energy under contract DE-AC52-06NA25396..

\section{References}

1. Hall, E.O., The Deformation and Ageing of Mild Steel: III Discussion of Results Proceedings of the Physical Society. Section B 1951. 64(9).

2. Petch, N.J., Cleavage strength of polycrystals. Iron and Science Institure-journal, 1953. 173.

3. Cottrell, A.H., Theory of brittle fracture in steel and similar metals. Metallurgical Society of American Institute of Mining, Metallurgical and Petroleum Engineers -- Transactions, 1958: p. 212.

4. Meyers MA, A.E., A model for the effect of grain size on the yield stress of metals. Philosophical Magazine A, 1982. 46: p. 737.

5. Adams, B.L., et al., Theory of grain boundary structure effects on mechanical behaviour. Materials Science Forum Proceedings of the 10th International Conference on Textures of Materials. Part 2 (of 2), Sep 20-24 1993, 1994. 157-6(pt 2): p. 1731-1738.

6. Tsurekawa, S., T. Tanaka, and H. Yoshinaga, Grain boundary structure, energy and strength in molybdenum. Materials Science and Engineering: A, 1994. 176(1-2): p. 341348.

7. Watanabe, T. and S. Tsurekawa, The control of brittleness and development of desirable mechanical properties in polycrystalline systems by grain boundary engineering. Acta Materialia, 1999. 47(15-16): p. 4171-4185.

8. Palumbo, G., et al., Grain boundary design and control for intergranular stresscorrosion resistance. Scripta Metallurgica et Materialia, 1991. 25(8): p. 1775-1780. 
9. Dahlberg, C.F.O., et al., A deformation mechanism map for polycrystals modeled using strain gradient plasticity and interfaces that slide and separate. International Journal of Plasticity, 2013. 43: p. 177-195.

10. Rohrer, G.S., et al., The distribution of internal interfaces in polycrystals. Zeitschrift für Metallkunde, 2004. 95(4): p. 197-214.

11. Tschopp, M.A. and D.L. McDowell, Asymmetric tilt grain boundary structure and energy in copper and aluminium. Philosophical Magazine, 2007. 87(25): p. 3871-3892.

12. Clark, W.A.T., et al., On the criteria for slip transmission across interfaces in polycrystals. Scripta Metallurgica et Materialia, 1992. 26(2): p. 203-206.

13. de Koning, M., et al., Modeling of dislocation-grain boundary interactions in FCC metals. Journal of Nuclear Materials, 2003. 323(2-3): p. 281-289.

14. Bieler, T.R., et al., The role of heterogeneous deformation on damage nucleation at grain boundaries in single phase metals. International Journal of Plasticity, 2009. 25(9): p. 1655-1683.

15. Asaro, R.J., Crystal Plasticity. Journal of Applied Mechanics, 1983. 50(4b): p. 921-934.

16. Kalidindi, S.R., C.A. Bronkhorst, and L. Anand, Crystallographic Texture Evolution in Bulk Deformation Processing of Fcc Metals. Journal of the Mechanics and Physics of Solids, 1992. 40(3): p. 537-569.

17. Kalidindi, S.R., Incorporation of deformation twinning in crystal plasticity models. Journal of the Mechanics and Physics of Solids, 1998. 46(2): p. 267-271.

18. Van Houtte, P., L. Delannay, and S.R. Kalidindi, Comparison of two grain interaction models for polycrystal plasticity and deformation texture prediction. International Journal of Plasticity, 2002. 18(3): p. 359-377.

19. Ng, K.S. and A.H.W. Ngan, Deformation of micron-sized aluminium bi-crystal pillars. Philosophical Magazine, 2009. 89(33): p. 3013-3026.

20. Kunz, A., S. Pathak, and J.R. Greer, Size effects in Al nanopillars: Single crystalline vs. bicrystalline. Acta Materialia, 2011. 59(11): p. 4416-4424.

21. Dietiker, M., et al., Deformation behavior of gold nano-pillars prepared by nanoimprinting and focused ion-beam milling. Acta Materialia, 2011. 59(5): p. 21802192.

22. Uchic, M.D., et al., Sample dimensions influence strength and crystal plasticity. Science, 2004. 305(5686): p. 986-989.

23. Fischer-Cripps, A.C., Nanoindentation. Mechanical Engineering Series, ed. F.F. Ling. 2002, New York: Springer.

24. Fischer-Cripps, A.C., Study of analysis methods of depth-sensing indentation test data for spherical indenters. Journal of Materials Research, 2001. 16: p. 1579-84.

25. Fischer-Cripps, A.C., Critical review of analysis and interpretation of nanoindentation test data. Surface and Coatings Technology, 2006. 200(14-15): p. 4153-4165.

26. Hay, J.L. and G.M. Pharr, Instrumented indentation testing. ASM Handbook.

27. Kalidindi, S.R. and S.J. Vachhani, Mechanical characterization of grain boundaries using nanoindentation. Current Opinion in Solid State and Materials Science, 2014. 18(4): p. 196-204.

28. Kalidindi, S.R. and S. Pathak, Determination of the effective zero-point and the extraction of spherical nanoindentation stress-strain curves. Acta Materialia, 2008. 56: p. 3523-32. 
29. Pathak, S., J. Shaffer, and S.R. Kalidindi, Determination of an effective zero-point and extraction of indentation stress-strain curves without the continuous stiffness measurement signal. Scripta Materialia, 2009. 60: p. 439-42.

30. Pathak, S., et al., Assessment of lamellar level properties in mouse bone utilizing a novel spherical nanoindentation data analysis method. Journal of the Mechanical Behavior of Biomedical Materials, 2012. 13(0): p. 102-117.

31. Pathak, S., et al., Viscoelasticity and high buckling stress of dense carbon nanotube brushes. Carbon, 2009. 47: p. 1969-1976.

32. Vachhani, S.J. and S.R. Kalidindi, Grain-scale measurement of slip resistances in aluminum polycrystals using spherical nanoindentation. Acta Materialia, 2015. 90(0): p. 27-36.

33. Pathak, S., et al., Studying grain boundary regions in polycrystalline materials using spherical nano-indentation and orientation imaging microscopy. Journal of Materials Science, 2012. 47(2): p. 815-823.

34. Zaefferer, S., et al., On the influence of the grain boundary misorientation on the plastic deformation of aluminum bicrystals. Acta Materialia, 2003. 51(16): p. 4719-4735.

35. Ma, A., F. Roters, and D. Raabe, On the consideration of interactions between dislocations and grain boundaries in crystal plasticity finite element modeling - Theory, experiments, and simulations. Acta Materialia, 2006. 54(8): p. 2181-2194.

36. Dalla Torre, F., et al., Grain Size, Misorientation, and Texture Evolution of Copper Processed by Equal Channel Angular Extrusion and the Validity of the Hall-Petch Relationship. Metallurgical and Materials Transactions A, 2007. 38(5): p. 1080-1095.

37. Kalidindi, S.R., A. Bhattacharya, and R. Doherty, Detailed Analysis of Plastic Deformation in Columnar Polycrystalline Aluminum Using Orientation Image Mapping and Crystal Plasticity Models. Proceedings of the Royal Society of London: Mathematical, Physical and Engineering Sciences., 2004. 460(2047 ): p. 1935 - 1956

38. Bhattacharyya, A., et al., Evolution of grain-scale microstructure during large strain simple compression of polycrystalline aluminum with quasi-columnar grains: OIM measurements and numerical simulations. International Journal of Plasticity, 2001. 17(6): p. 861-883.

39. Pathak, S., et al., Studying grain boundary regions in polycrystalline materials using spherical nano-indentation and orientation imaging microscopy. Journal of Materials Science, 2012. 47(2): p. 815-823.

40. Tabor, D., The hardness of metals. 1951: Clarendon Press, Oxford University Press. ix + 176.

41. Bucaille, J.L., et al., Determination of plastic properties of metals by instrumented indentation using different sharp indenters. Acta Materialia, 2003. 51(6): p. 1663-1678.

42. Rho, J.-Y., T.Y. Tsui, and G.M. Pharr, Elastic properties of human cortical and trabecular lamellar bone measured by nanoindentation. Biomaterials, 1997. 18(20): p. 1325-1330.

43. Poole, W.J., M.F. Ashby, and N.A. Fleck, Micro-hardness of annealed and workhardened copper polycrystals. Scripta Materialia, 1996. 34(4): p. 559-564.

44. Oliver, W.C. and G.M. Pharr, Improved technique for determining hardness and elastic modulus using load and displacement sensing indentation experiments. Journal of Materials Research, 1992. 7(6): p. 1564-1580. 
45. Oliver, W.C. and G.M. Pharr, Measurement of hardness and elastic modulus by instrumented indentation: Advances in understanding and refinements to methodology. Journal of Materials Research, 2004. 19: p. 3-20.

46. $\mathrm{Li}, \mathrm{X}$. and B. Bhushan, A review of nanoindentation continuous stiffness measurement technique and its applications. Materials Characterization, 2002. 48(1): p. 11-36.

47. Pathak, S., D. Stojakovic, and S.R. Kalidindi, Measurement of the local mechanical properties in polycrystalline samples using spherical nanoindentation and orientation imaging microscopy. Acta Materialia, 2009. 57: p. 3020-8.

48. Hertz, H., Miscellaneous Papers. New York: MacMillan and Co., Ltd, 1896.

49. Johnson, K.L., Indentation Contact Mechanics. 1985: Cambridge University Press, Cambridge.

50. Sneddon, I.N., Relation between load and penetration in axisymmetric Boussinesq problem for punch of arbitrary profile. International Journal of Engineering Science, 1965. 3: p. 47-57.

51. Vachhani, S.J., R.D. Doherty, and S.R. Kalidindi, Effect of the continuous stiffness measurement on the mechanical properties extracted using spherical nanoindentation. Acta Materialia, 2013. 61(10): p. 3744-3751.

52. Pathak, S., et al., Importance of surface preparation on the nano-indentation stress-strain curves measured in metals. Journal of Materials Research, 2009. 24: p. 1142-55.

53. Gonzalez, D., et al. Modelling \& measurement of plastic deformation \& grain rotation in $3 D$ at the grain to grain level. in Proceedings of the 1st World Congress on Integrated Computational Materials Engineering (ICME). 2011. John Wiley \& Sons.

54. Gene Simmons, H.W., Single Crystal Elastic Constants and Calculated Aggregate Properties. 1971, Cambridge MIT Press.

55. Vlassak, J.J. and W.D. Nix, Measuring the elastic properties of anisotropic materials by means of indentation experiments. Journal of the Mechanics and Physics of Solids, 1994. 42(8): p. 1223-1245.

56. Metallography and Microstructures, in ASM handbooks Online, G.F.V. Voort, Editor. 2004.

57. $\quad$ Bunge, H., Texture Analysis in Materials Science. Butterworths, 1982.

58. Bunge, H.-J., Texture analysis in materials science. Mathematical Methods. 1993, Göttingen: Cuvillier Verlag.

59. Gonzalez, D., et al., Modelling the effect of elastic and plastic anisotropies on stresses at grain boundaries. International Journal of Plasticity, 2014. 61: p. 49-63.

60. Sun, S., B.L. Adams, and W.E. King, Observations of lattice curvature near the interface of a deformed aluminium bicrystal. Philosophical Magazine A: Physics of Condensed Matter, Structure, Defects and Mechanical Properties, 2000. 80(1): p. 9-25.

61. Anthony D. Rollett, A.P.B., C.G. Roberts, An Overview of Accomplishments and Challenges in Recrystallization and Grain Growth. Materials Science Forum, 2007. 558559: p. 33-42.

62. Pouillier, E., et al., A study of intergranular fracture in an aluminium alloy due to hydrogen embrittlement. International Journal of Plasticity, 2012. 34: p. 139-153. 